\title{
Credit chains and bankruptcy propagation in production networks
}

\author{
Stefano Battiston ${ }^{\mathrm{a}, *}$, Domenico Delli Gatti ${ }^{\mathrm{b}}$, Mauro Gallegati ${ }^{\mathrm{c}}$, \\ Bruce Greenwald $^{\mathrm{d}}$, Joseph E. Stiglitz ${ }^{\mathrm{d}}$ \\ ${ }^{a}$ Chair of Systems Design, ETH Zurich, Kreuzplatz 5, 8032 Zurich, Switzerland \\ ${ }^{\mathrm{b}}$ Institute of Quantitative Methods and Economic Theory, Catholic University of Milan, Largo Gemelli 1, \\ 20123 Milan, Italy \\ ${ }^{\mathrm{c}}$ Department of Economics, Universitá Politecnica delle Marche, Piazz.le Martelli 8, 60121 Ancona, Italy \\ ${ }^{\mathrm{d}}$ Columbia Business School, Columbia University, Uris Hall, 3022 Broadway, New York, NY 10027, USA
}

Available online 29 March 2007

\begin{abstract}
We present a simple model of a production network in which firms are linked by supplier-customer relationships involving extension of trade-credit. Our aim is to identify the minimal set of mechanisms which reproduce qualitatively the main stylized facts of industrial demography, such as firms' size distribution, and, at the same time, the correlation, over time and across firms, of output, growth and bankruptcies. The behavior of aggregate variables can be traced back to the direct firm-firm interdependence. In this paper, we assume that the number of firms is constant and the network has a periodic static structure. But the framework allows further extensions to investigate which network structures are more robust against domino effects and, if the network is let to evolve in time, which structures emerge spontaneously, depending on the individual strategies for orders and delivery.
\end{abstract}

(C) 2007 Elsevier B.V. All rights reserved.

JEL classification: E32; D85; L14; L22

Keywords: Network; Supply chain; Credit chain; Financial contagion; Bankruptcy

\footnotetext{
${ }^{*}$ Corresponding author. Tel.: + 41446328464 ; fax: + 41446321880.

E-mail address: sbattiston@ethz.ch (S. Battiston).

$U R L:$ http://www.sg.ethz.ch/people/sbattiston (S. Battiston).
}

0165-1889/\$ - see front matter (C) 2007 Elsevier B.V. All rights reserved. doi:10.1016/j.jedc.2007.01.004 


\section{Introduction}

The basic framework in mainstream economic theory, i.e., the Arrow-Debreu general equilibrium model, rests on the assumption that individuals take decisions in isolation - they play a 'game against nature' - using only the information contained in a general market signal such as the price vector. In such a framework, interaction and co-ordination occur only indirectly through prices. Direct interaction among agents is ruled out by construction. Moreover, exchange takes place only when equilibrium prices have been discovered so that out-of-equilibrium dynamics due to exchange at 'false prices' are ruled out by assumption and individual choices are always consistent: the failure of co-ordination which is likely to arise in a decentralized market economy is simply assumed away (Leijonhuvfud, 1992, 1993; Hahn and Solow, 1995).

Prices are undoubtedly a fundamental part of the picture, but the price mechanism can work well only if information is perfect and markets are complete. If this is not the case, i.e., if the future is uncertain, we cannot ignore direct interactions which assume the form of co-ordination mechanisms that arise in spatio-temporal way - i.e., supply chains, communication, imitation, learning, trust and credit relationships. Credit is extended by one firm to another (trade credit), by one bank to another (interbank credit) and by banks to firms (loans). In other words, there is a network of production and credit relationships among firms, among banks and between firms and banking system. In this context, complex patterns of heterogeneous agents' interactions at the microlevel lead to the emergence of statistical regularity at the macroeconomic level through a self-organized process.

The structure and macroeconomic impact of a production network has been studied for instance by Bak et al. (1993). As for credit, the role of bank loans has been extensively dealt with in the enormous literature on firms-banks relationships (for an overview of the main issues, see Stiglitz and Greenwald, 2003). An interesting new line of research has emphasized the role of interbank credit in determining phenomena of financial contagion (Allen and Gale, 2000). Last but not least, trade credit is an important part of the network of credit relationships. In the U.S., trade credit represented one-half of the short term liabilities of the corporate sector in 2004 (Boissay, 2006). Moreover, trade credit is largely used as collateral in bank borrowing, especially by small and medium sized firms. In the U.S., lines of credit secured by accounts receivables represented approximately one quarter of total bank loans in 1998 (Klapper, 2001). In Italy, loans secured by receivables were $22 \%$ of total loans and 54\% of short term loans in 2002 (Omiccioli, 2005). In the theoretical literature, Kiyotaki and Moore (1997) emphasize the role of trade credit as a propagation mechanism. So far, however, the issue has been underresearched, both at the theoretical and empirical levels, with the notable exception of Boissay (2006). ${ }^{1}$

The focus of the present paper is on the role of firms' network in determining macroeconomic outcomes such as the agglomeration over time of production

\footnotetext{
${ }^{1}$ See also Petersen and Rajan (1997), Elligsen and Burkart (2002), Lee and Stowe (1993).
} 
activity and/or of bankruptcies. We explore the features of a networked economy in which $N$ firms are organized in $M$ production levels. Each firm at a certain level is supplied by a subset of firms in the upper level (suppliers) and supplies a subset of the firms in the lower level (customers). In other words, the firm is a customer (of suppliers located in the upper level) and a supplier (of customers located in the lower level) at the same time. The bottom level consists of retailers, i.e., firms that sell in the consumer market. The top level consists of firms that provide primary goods to the other firms. Firms are connected by means of two mechanisms: (i) the output of supplier firms is an input for customer firms; (ii) supplier firms extend trade credit to customers.

In each period, firms in the bottom level (retailers) determine their desired output on the basis of the demand coming from the consumers and their production capacity, and send orders to the upper level. Firms in the upper level, in turn, determine their desired output on the basis of the demand coming from their customers (i.e., the retailers). In sequence one after another, all production levels do the same, up to the first level. At this point, production starts in the first level and proceeds one level after the other according to the established production plans. When production reaches the bottom level, products are sold to the consumers and a sequence of payments is activated going from the final consumers to retailers, from retailers to producers and so on up to the providers of primary goods.

At each level, in each period, a firm as a supplier is paid for its product by its customers and only afterwards pays the cost of inputs to the suppliers as a customer. In a sense, therefore, the suppliers extend trade credit to the firm for an amount equal to $100 \%$ of the cost of supplies. If cost exceeds the proceeds from sales, the firm goes bankrupt and does not pay its suppliers. In this case, the firm stops production and is replaced by a new firm (so that the number of firms remains constant). The trade credit contract is only implicitly sketched: we neither design the optimal trade credit scheme nor look for the optimal amount of trade credit a customer firm should require. These issues are left to further extensions and developments of the present framework. In this paper, we focus instead on different strategies that firms can follow to allocate credit to suppliers and fulfill orders from customers. Weisbuch and Battiston (2007) have recently studied another model of production network developed in a similar framework. Their model is somehow simpler, as it does not include credit, and it is investigated in relation to geographical economics issues.

In our model, if a firm is unable to reimburse debt, it goes bankrupt. In principle, bankruptcy can be of two types. Bankruptcy of the first type may be triggered by an unexpected shock to revenues or to costs so that average revenue turns out to be 'too low' with respect to average cost. In this paper, we model the unexpected shock to revenues along the lines of Greenwald and Stiglitz (1993) as a bad realization of the (random) relative price of the firm. Bankruptcy of the second type occurs when a supplier at a certain level is not paid by the customer at the lower level. This second type of bankruptcy can be dealt with only in the context of network economies such as the one considered in the present paper. In our context, the failure to fulfill debt 
commitments by a customer may hamper the solvency of the supplier, who may become unable in turn to pay its own suppliers located in the upper level, which may lead to a chain of similar failures (domino effect) and in extreme cases result in bankruptcy avalanches. When a firm goes bankrupt, in fact, the probability of bankruptcy in connected firms increases, yielding clustered fluctuations in the number of failing firms. In other words, a single bankruptcy (of the first type) may have systemic repercussions through an avalanche of bankruptcies of the second type.

Previous multi-agent models of financial fragility (Delli Gatti et al., 2005) have been able to reproduce stylized facts of firms' demography and aggregate output in a framework in which firms go bankrupt because of unexpected price or cost fluctuations. However, such models incorporate only the indirect interaction among firms that takes place through the endogenous determination of the interest rate on bank loans. Notice that in this case, a single bankruptcy of the first type - due, for instance, to an unexpected negative shock to revenues - may have systemic repercussions. In Delli Gatti et al. (2005), in fact, the banking system reacts to the above-mentioned bankruptcy of the first type by restraining the supply of credit and pushing up the interest rate. The increase in the interest rate may trigger and avalanche of bankruptcies. These avalanches, however, are of the first type, i.e., they are due to an unexpected cost push engendered by the interest rate hike.

Such an approach is similar to the 'mean field approximation' in statistical physics, which consists in supposing that each unit of the system is interacting with an average unit. The mean field approximation yields useful predictions when units interact in an all-to-all fashion and are not too heterogeneous; otherwise, the dynamics of the system may be qualitatively different from the mean field prediction. In the present framework, we emphasize the role of direct local interaction among firms in the generation of bankruptcy avalanches. In a production network, interactions are local and units are highly heterogeneous indeed.

It is worth mentioning that in (Delli Gatti et al., 2005) the evolution of the net worth of firms can be very roughly approximated as a set of multiplicative stochastic processes (MSP). The results on the firms' size distribution are then consistent with the well known fact that power law distributions can be obtained by combining an MSP with a specific additional ingredient - either a reflecting barrier or additive noise or a stochastic resetting of the size (Kesten, 1973; Biham et al., 1998; Sornette, 1998; Nirei and Souma, 2003). Also in the model presented in this paper, it is possible to show that the evolution of the net worth of firms is related to a set of MSP. However, this issue will be developed in future work.

Overall, we proceed by identifying a minimal set of mechanisms that reproduce qualitatively the main facts of firm demography and the emergence of spatiotemporal patterns for output, growth and failures. The paper is organized as follows. In Section 2 we first describe a framework in which a class of models can be developed. We then provide a detailed description of a specific model, pointing out limitations and simplifications. In Section 3 we discuss the mechanisms of failure propagation and in Section 4 we report the results of our computer simulations. Conclusions are presented in Section 5. 


\section{The model}

We first outline the organization of the production network (Section 2.1) and the temporal structure of the events (Section 2.2). We then describe in detail the model (Sections 2.4-2.9).

\subsection{Economic environment}

The economy consists of $N$ firms organized in $M$ production levels. We will denote firms with indices $i, j, k, l, \ldots$ and levels with indices $J, K, L, \ldots$ We adopt the convention that production takes place along the vertical axis in downwards direction. The structure of the connections defines the production network as in the example shown in Fig. 1, in which arrows represent supply of goods (supply proceeds downwards, while money moves upwards).

Each firm in a level $K$ is supplied by a subset of firms in the upper level $K-1$ and in turn supplies a subset of the firms in the lower level $K+1$. The bottom level $K=M$ represents firms that sell in the consumer market (retailers). The top level $K=1$ represents primary producers. Firms are connected to each other through two mechanisms:

1. A firm asks for inputs from the suppliers in order to produce output.

2. A firm asks for payments from the customers in order to realize profit.

The output of each level $K$ is produced by processing the input from the previous level $K-1$. Output is qualitatively different from input. For the sake of simplicity, we assume the following linear technology:

$$
Y_{i}^{(K)}=\sum_{j \in V_{i}^{S}} Q_{i j}^{(K, K-1)} Y_{j}^{(K-1)},
$$

where $Y_{i}$ is the output of firm $i, S_{i}$ is the set of suppliers of firm $i$, and $Q_{i j}$ represents the fraction of the total output of firm $j$ that firm $i$ uses to produce its own output.

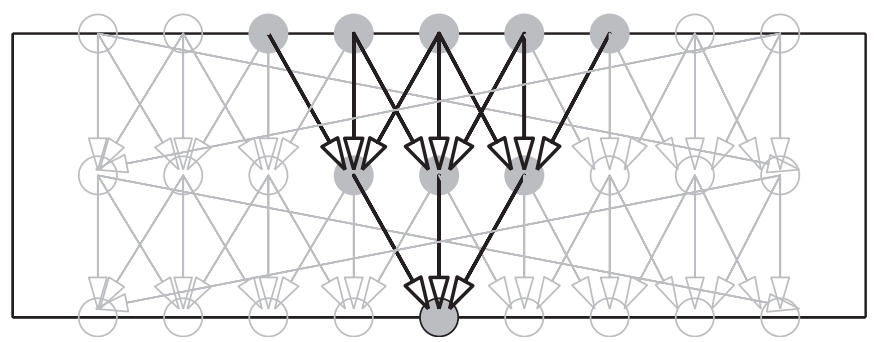

Fig. 1. Example of structure for the production network. The direction of production is from top to bottom. Each firm in a level receives goods from a subset ( 3 in this case) of firms from the upper level. The top level consists of primary producers. Layer 3 (from the top) consists of firms that sell in the consumer market (retailers). We have highlighted in dark gray the set of all suppliers upward from a given retailer. 
In other words, $Q$ is the input-output matrix and for any $K$, it follows that

$$
\sum_{i \in \text { level } K} Q_{i j}^{(K, K-1)}=1 \quad \forall j \in \text { level } K-1 .
$$

\subsection{Timing}

We have to model the fact that over time, firms decide their desired amount of production, send orders, produce, deliver to customers and pay suppliers. We assume that time is discrete and divided into periods, each period including the following events for all firms: at the beginning of each period (or time step) $t$, orders flow upwards; then production and delivery flow downward. At the end of the period, money flows upward.

In greater detail, at the beginning, all firms in the bottom level $M$ determine their desired output, based on the demand they face on the market and their production capacity, and then send orders to the upper level $M-1$. Afterwards, all firms in level $M-1$ determine their desired output based on the demand they face from their customer firms in level $M$. One after another, all levels do the same, up to level 1 (primary producers). Once the desired output is known, firms can compute their expected output (see Section 2.8), based on the expected output of the suppliers, which they communicate to the firms downward. This allows customer firms to allocate the necessary resources and premises to process the inputs they will receive.

At this point, production starts in level 1 and proceeds downward one level after the other, as each firm needs the input from its suppliers in order to produce. Output produced by a firm is delivered to customers on the basis of full trade credit; we rule out the possibility of inventory accumulation. When production reaches the bottom level, products are fully sold in the consumer market.

At the end of the period, a sequence of payments proceeds upwards from the retailers up to the primary producers. At each level, each firm pays its suppliers upstream only after having been paid by its customers. If costs exceeds revenues, the firm goes bankrupt and does not pay the suppliers in the current period. Moreover, the firm stops production for a number $\tau$ of periods in the future, after which it is replaced by a new firm endowed with an assigned initial value of production capacity. During those $\tau$ periods, the suppliers of that firm do not receive orders from it, nor do the customers receive production from it. Therefore, bankruptcy at the end of period $t$ results not only in disruption of payments but also in a temporary local disruption in the production chain which is repaired in period $t+\tau+1$.

\subsection{Remarks}

The structure of the connections does not change during the process. This means that when a firm goes bankrupt, its customers do not create new links with other suppliers. This follows from the assumption of prohibitively high costs of establishing relations with new suppliers. So far, we have described a general framework, while the mechanisms involved can be specified in several ways 
(for example, we have to specify the dynamics of price, profit and net worth). However, some of the results presented in this paper do not depend on the specification of such mechanisms. Therefore, the present structure is a candidate for a class of models sharing similar behavior, in particular, concerning the conditions for the occurrence of avalanches of bankruptcies which are analyzed in Section 3. In the following, we provide a detailed description of a simple version of the model and a discussion of its limitations. In any period $t$ each firm $i$ is endowed with a level of real net worth $A_{i}(t)$, defined as the stock of the firm's assets in real terms, that has been financed only through net profits (we assume complete equity rationing).

\subsection{Desired output}

Firm $i$ at level $K$ determines at time $t$ its desired output, $Y_{i}^{(\mathrm{d}, K)}$. This depends on the orders received from level $K+1$, with the constraint of its production capacity that we assume to be proportional to net worth $A_{i}^{(K)}$ by a constant $\theta>0$ (as stated in Eq. (3)). Therefore, capacity is financially constrained as, for instance, in Greenwald and Stiglitz (1993) and in related work by Delli Gatti et al. (2005). As in Greenwald and Stiglitz we can conceive of $\theta A_{i}^{(K)}(t)$ as the optimal (i.e., maximizing expected profit) output in the presence of bankruptcy costs.

Hence, desired output is defined as follows:

$$
Y_{i}^{(\mathrm{d}, K)}(t)=\min \left\{\theta A_{i}^{(K)}(t), \sum_{j \in V_{i}^{C}} O_{i j}^{(K, K+1)}(t) Y_{j}^{(\mathrm{d}, K+1)}(t)\right\} .
$$

In the equation above, $V_{i}^{C}$ is the set of customers of firm $i, O^{(K, K+1)}$ is the order matrix describing the orders from level $K+1$ to $K$, and in particular $O_{i j}^{(K, K+1)}$ is the fraction of the total supply needed by firm $j$ that firm $j$ orders to firm $i$. In matrix notation we can write

$$
Y^{(\mathrm{d}, K)}(t)=\min \left\{\theta A^{(K)}(t), O^{(K, K+1)} Y^{(\mathrm{d}, K+1)}(t)\right\} .
$$

For level $M$, we assume that at each time step the consumer market absorbs the whole production and therefore

$$
Y^{(\mathrm{d}, M)}(t)=\theta A^{(K)}(t) .
$$

\subsection{Expected and effective output}

Once the desired output is known at all levels, firms compute their expected output, based on the expected output of the suppliers. Here, 'expected' has nothing to do with 'expectation value' in statistical sense. A firm $i$ may not be able to fulfill the orders of its customers, either because they exceed its production capacity or because the input from its suppliers is insufficient. As a result, supply can be smaller than the ordered quantity and therefore the expected output of firm $i, Y_{i}^{(\mathrm{e})}$, can be smaller than the desired one $Y_{i}^{(\mathrm{d})}$. In this version of the model, firms have a fixed set of suppliers (the network structure is static) and they cannot look for new suppliers. However, 
there is some freedom in the way firms decide to place orders to their suppliers, in other words, the ways $O_{i j}^{(K, K+1)}$ are determined. This is discussed later on and plays an important role. The production function of firms is assumed to be linear so that the output of a firm in level $K$ is a linear combination of the input received from the suppliers in level $K-1$. This yields

$$
\begin{aligned}
& Y_{i}^{(\mathrm{e}, 1)}(t)=Y_{i}^{(\mathrm{d}, 1)}(t) \\
& Y_{i}^{(\mathrm{e}, K)}(t)=\sum_{j \in V_{i}^{S}} Q_{i j}^{(K, K-1)}(t) Y_{j}^{(\mathrm{e}, K-1)}(t) .
\end{aligned}
$$

For firms at level 1, the expected output coincides with the desired one, as they do not have suppliers. $V_{i}^{S}$ is the set of suppliers of firm $i, Q^{(K, K-1)}$ is the input-output matrix describing the transformation of input from level $K-1$ into the output of level $K$. Each entry $Q_{i j}^{(K, K-1)}$ represents the fraction of the total output of firm $j$ that firm $i$ uses to produce its own output. Firms in level 1 are primary producers and do not need any supply, therefore $Y_{i}^{(\mathrm{e}, 1)}=Y_{i}^{(\mathrm{d}, 1)}$. In matrix notation, the output of any level can be expressed as a function of the output of the first level as follows:

$$
Y^{(\mathrm{e}, K)}(t)=Q^{(K, K-1)}(t) Y^{(\mathrm{e}, K-1)}(t)=Q^{(K, K-1)}(t) \cdot \ldots \cdot Q^{(2,1)}(t) Y^{(\mathrm{e}, 1)}(t) .
$$

The expected output is communicated downward to customers. Any two firms engaged in a supplier-customer relation agree on this amount to be delivered and paid at the end of the period. Customer firms allocate the necessary resources and premises to process the expected input they will receive from suppliers.

At this point, we include in the model some occasional production failures (due, for instance, to technical problems). At each period $t$, with probability $q$, the production of firm $i$ is lost during the processing and no output is delivered to customers. This event occurs independently of the financial state of firms $i$ and this failure lasts only one period. Therefore, we have to rewrite the effective output of $i$, $Y_{i}^{(K)}(t)$, as

$$
Y_{i}^{(K)}(t)=Y_{i}^{(\mathrm{e}, K)}(t) S_{i}(t)
$$

where $S_{j}(t)=0$ with probability $q$ and $S_{j}(t)=1$ with probability $1-q$.

\subsection{Production costs}

The output produced by firm $i$ is sold to the customer at the price $P_{i}(t)$ (no inventory accumulation). We can think of the price of a firm's output in level $K$ as $P_{i}(t)=P^{(K)}(t) u_{i}(t)$ where $P^{(K)}(t)$ is the general price at level $K$ and $u_{i}(t)$ is the relative price for the output of the single firm. We assume that $u_{i}(t)$ is a random variable, uniformly distributed in $\left[1-\delta_{P}, 1+\delta_{P}\right]$ and independent of $P^{(K)}(t)$. Therefore, firm $i$ incurs the following cost to get its supply of inputs from level $K-1$ :

$$
\tilde{C}_{i}^{(\mathrm{s}, K)}(t)=\sum_{j \in V_{i}^{S}} Q_{i j}^{(K, K-1)} P^{(K-1)}(t) u_{j}(t) Y_{j}^{(K-1)} .
$$


The cost of inputs in real terms is obtained by dividing nominal costs by the level of prices in the level $K$ :

$$
\begin{aligned}
C_{i}^{(\mathrm{s}, K)}(t) & =\frac{P^{(K-1)}(t)}{P^{(K)}(t)} \sum_{j \in V_{i}^{S}} Q_{i j}^{(K, K-1)}(t) u_{j}(t) Y_{j}^{(K-1)}(t) \\
& =c_{\mathrm{s}} \sum_{j \in V_{i}^{S}} Q_{i j}^{(K, K-1)}(t) u_{j}(t) Y_{j}^{(K-1)}(t)
\end{aligned}
$$

where $c_{\mathrm{s}}$ is defined as the ratio of the price levels at level $K-1$ and $K$ and we assume it to be the same for all $K$.

Firm $i$ also incurs a cost associated with the resources used in processing the input (labor and premises). As for the supply cost, this cost is assumed to be proportional to the expected output through a constant $c_{\mathrm{r}}>0$. We assume that the resources allocated by the customers of $i$ to process its expected output cannot be dis-allocated within the current time period. Therefore, in case of a production failure of $i$, its customers run a cost proportional to the expected output and not to the effective output:

$$
C_{i}^{(\mathrm{r}, K)}(t)=c_{\mathrm{r}} Y_{i}^{(\mathrm{e}, K)}=c_{\mathrm{r}} \sum_{j \in V_{i}^{S}} Q_{i j}^{(K, K-1)}(t) Y_{j}^{(\mathrm{e}, K-1)}(t) .
$$

Of course, in the case of a production failure by $i$, the customers of $i$ do not incur any supply cost. On the other hand, firm $i$ not only does not receive any payment but has also to pay for the input from its suppliers. The production of firm $i$ resumes at the next time step, if it has survived the shock. In conclusion, the production cost of firm $i$ is the sum of the two terms defined above:

$$
C_{i}^{(K)}(t)=C_{i}^{(\mathrm{s}, K)}(t)+C_{i}^{(\mathrm{r}, K)}(t) .
$$

\subsection{Profit and bankruptcy}

In each period, when output is sold in the consumer market and payments start, some firms may realize sales revenue smaller than their supply costs. If this loss is high enough, firms go bankrupt and do not pay their suppliers. Therefore, we have to distinguish between the output delivered by firm $i$ to its customers, $Y_{i}(t)$, and the output $Y_{i}^{\mathrm{s}}(t)$ that is actually paid for ('s' for 'sold'), at price $u_{i}(t)$, to firm $i$ by its customers. Profit in real terms is equal to the difference between revenues and costs in real terms:

$$
\pi_{i}^{(K)}(t)=u_{i}(t) Y_{i}^{(\mathrm{s}, K)}(t)-C_{i}^{(K)}(t) .
$$

Profit, which can be negative or positive, incrementally changes the real net worth of the firm:

$$
A_{i}^{(K)}(t+1)=\rho A_{i}^{(K)}(t)+\pi_{i}^{(K)}(t),
$$

where $1-\rho$ measures a depreciation rate. 
We assume that firms go bankrupt when the ratio of profit and net worth becomes smaller than a negative threshold value:

$$
\frac{p i_{i}^{(K)}}{A_{i}^{(K)}}<-\beta
$$

with $1>\beta>0$. If a firm goes bankrupt at time $t$, it stops supplying customers and paying suppliers for a number $\tau$ of time steps (referred to as 'inactivity time' in the following). During these time steps, neighboring firms are not allowed to look for alternative customers or suppliers, as the network structure is static. Firms can however (at least in some of the scenarios considered in the following) adjust their orders as a function of the production capacity of the suppliers. As this is proportional to net worth, it means that customers order less and less when a supplier's net worth decreases. Once the inactivity time elapsed, the bankrupt firm is replaced by a new firm with the same links as its predecessor and its net worth is re-initialized: $A(t+\tau+1)=A_{\text {entry }}$.

\subsection{Responsiveness of firms to supply failure}

Whenever a supplier $j$ unexpectedly does not deliver its input to firm $i$, the firm may not be able to reduce immediately the resources (labor and premises) that it had allocated to process such input. This depends of course on the labor regulations of the country and the industrial sector. In this paper, we shall consider only two simple scenarios at the extreme ends of the range of responsiveness of the firm. Suppose, for instance, that the supplier $j$ goes bankrupt at time $t$ and does not deliver any input to $i$ at time steps $t+1, \ldots, t+\tau$. If firm $i$ is not able to adapt its resources to the new input, then at time steps $t+1, \ldots, t+\tau$ it will incur a cost proportional to the input it received from $j$ at time $t$. If the firm is adaptive, it will not run any cost in relation to $j$.

In conclusion, in the first scenario, the costs associated with resources are

$$
C_{i}^{(\mathrm{r}, K)}(t)=c_{\mathrm{r}} \sum_{\substack{j \in V_{i}^{S} \\ j \text { bankrupt }}} Q_{i j}^{(K, K-1)} Y_{j}^{(K-1)}\left(t^{\prime}\right)+\sum_{\substack{l \in V_{i}^{S} \\ l \text { active }}} Q_{i l}^{(K, K-1)} Y_{l}^{(\mathrm{e}, K-1)}(t),
$$

where $t^{\prime}$ is the time step at which $j$ went bankrupt (the last time). In the second scenario, it holds Eq. (11).

\subsection{Strategies for placing orders and delivery}

Although the network is static in this version of the model, and therefore the set of suppliers of a firm is fixed, still there are many possible ways to allocate orders to the suppliers. Consistently with our bounded rationality framework, we consider two simple strategies for placing orders and one strategy for delivering.

1. Firm $i$ places orders evenly across suppliers:

$$
O_{i j}^{(K, K+1)}(t)=\frac{1}{\left|V_{i}^{S}\right|},
$$


where $\left|V_{i}^{S}\right|$ is the cardinality of the set of suppliers of firm $i$ (notation is consistent with Eq. (3)).

2. Firm $i$ places orders proportionally to the production capacity of suppliers:

$$
O_{i j}^{(K, K+1)}(t)=\frac{A_{j}(t)}{\sum_{k \in V_{i}^{S}} A_{k}(t)} .
$$

Strategy 1 stimulates the growth of all the suppliers of a firm and tends to keep a balanced portfolio of suppliers, while strategy 2 favors the largest supplier, so that in the long run firms tend to have only one main supplier.

As explained in Section 2.5, in our simple setting it may occur that a supplier cannot fulfill the demands of its customers. In this case, several ways of allocating the delivery are possible and here we consider the following simple strategy:

1. Firm $j$ delivers to each customer $l$ in proportion to its order, relative to the orders of the other customers:

$$
Q_{i j}^{(K, K-1)}(t)=\frac{O_{i j}^{(K, K+1)}(t) Y_{j}^{(K-1)}(t)}{\sum_{l \in V_{j}^{C}} O_{l j}^{(K, K+1)}(t) Y_{j}^{(K-1)}(t)} .
$$

The equation above satisfies the condition of Eq. (2).

\section{Analysis of the model}

\subsection{Growth}

In this section we discuss the conditions that result in positive average growth across firms. For sake of simplicity, for this approximated calculation, we consider a two-layer network and we assume that prices are fixed $\left(u_{i}(t)=1 \forall i, t\right)$.

We can then write the supply cost for firm $i$ as

$$
C_{i}^{(\mathrm{s}, K)}(t)=c_{\mathrm{s}} \sum_{j \in V_{i}^{S}} Q_{i j}^{(K, K-1)}(t) Y(t)_{j}^{(K-1)}=c_{\mathrm{s}} Y(t)_{i}^{(K)} .
$$

Because each supplier $j$ of firm $i$ fails with probability $q$, its output is, on average, $Y_{j}^{(K-1)}=(1-q) Y_{j}^{(\mathrm{e}, K-1)}$. Using this relation, we rewrite the resource cost (Eq. (11)) as

$$
C_{i}^{(\mathrm{r}, K)}(t)=c_{\mathrm{r}} \sum_{j \in V_{i}^{S}} Q_{i j}^{(K, K-1)}(t) \frac{1}{(1-q)} Y_{j}^{(K-1)}(t) .
$$

This is a mean field approximation, in which we have replaced a fluctuating term with its average. When demand equals (or exceeds) supply (see Eq. (3)), we have $Y_{i}^{(K)}(t)=\theta A_{i}(t)^{(K)}$ (where $\theta$ can be assumed to equal 1 without loss of generality). 
Therefore plugging the expressions above for supply cost and resource cost in Eqs. (12) and (13), we can rewrite Eq. (14) as

$$
A_{i}(t+1)^{(K)}=\rho A_{i}(t)^{(K)}+\pi_{i}(t)^{(K)}=\left(\rho+1-c_{\mathrm{s}}+\frac{c_{\mathrm{r}}}{(1-q)}\right) A_{i}(t)^{(K)} .
$$

From this expression, it follows that the average growth of firms, defined as

$$
g(t)=\frac{1}{N} \sum_{i} \frac{A_{i}(t+1)-A_{i}(t)}{A_{i}(t)}
$$

is positive if

$$
\rho+1-c_{\mathrm{s}}+\frac{c_{\mathrm{r}}}{(1-q)}>1 \rightarrow \rho>c_{\mathrm{s}}+\frac{c_{\mathrm{r}}}{(1-q)} .
$$

Not surprisingly, the condition requires that the sum of the parameters related to cost is smaller than the depreciation factor. Let us now consider the aggregate growth, $G(t)$, defined as

$$
G(t)=\frac{\sum_{i, K} A_{i}^{(K)}(t+1)-\sum_{i, K} A_{i}^{(K)}(t)}{\sum_{i, K} A_{i}^{(K)}(t)} .
$$

Notice that $G(t)$ is not the same as $g(t)$. Moreover, as discussed in the results sections, the model generates heterogeneous firm size, which does not follow Gaussian statistics (not even in log scale). As a consequence, it is not possible to easily deduce a condition for the aggregate growth, $G(t)$, to be positive. For instance, if most firms have negative growth but the few largest firms have positive growth, it is possible to have negative average growth of firms (Eq. (23)) and yet positive growth in the aggregate (Eq. (25)).

\subsection{Propagation of bankruptcies}

In this section we want to make clear the distinction between the effect of a bankruptcy on the output of suppliers/customers and its effect on the probability of bankruptcy of these firms. A bankruptcy, or simply the change in output of a firm, affects the output of its customers (because they need its input to produce). However, their probability of going bankrupt is not necessarily increased. Let us introduce the notion of bankruptcy avalanche. By avalanche of events $B$ we mean a process in which one event of type $B$ at time $t$ in firm $i$ determines with a certain probability an event of the same type in each of the connected firms. This implies that after a few time steps, a number $a$ of events $B$ has occurred ( $a$ is also called the 'size' of the avalanche).

The production network modelled in this paper is represented by a directed graph in which the direction of the edges follows the direction of production. However, bankruptcies can propagate in the same direction as production or in the opposite one. We will speak of downward or upward propagation, respectively. If bankruptcies can propagate only downward/upward, when they reach the bottom/top level they 
stop, as shown in Fig. 2(a,b). In this case, the only firms affected are those in the cone downward/upward of the initial bankruptcies.

If bankruptcies can propagate simultaneously in both directions, then, and only then, are they 'reflected' diagonally at each level and the result is a net horizontal propagation that is perpendicular to the direction of production (Fig. 2(c,d)). In the following we will speak of horizontal bankruptcy propagation to mean the situation in which bankruptcies can propagate potentially to the whole network and not only to the downward/upward cone of firms.

In our model, bankruptcies occur when the ratio of profit and net worth becomes smaller than a negative threshold, $\pi_{i} / A_{i}<-\beta$. If the real price of sale for firm $i, u_{i}(t)$, is a stochastic variable with the probability distribution function $\mu\left(u_{i}\right)$, we can write the probability $\mathscr{P}_{i}^{\mathrm{B}}$ of bankruptcy for firm $i$ as follows (to keep notation simple, the level $K$ of the firm is not indicated):

$$
\begin{aligned}
\mathscr{P}_{i}^{\mathrm{B}} & =\mathscr{P}\left\{\pi_{i}(t)<-\beta A_{i}(t)\right\} \\
& =\mathscr{P}\left\{u_{i}(t) Y_{i}^{\mathrm{s}}(t)-C_{i}(t)<-\beta A_{i}(t)\right\} \\
& =\mathscr{P}\left\{u_{i}(t)<\frac{-\beta A_{i}(t)+C_{i}(t)}{Y_{i}^{\mathrm{s}}(t)}\right\}=\int_{1-\delta}^{u_{i}^{*}(t)} \mu(u) \mathrm{d} u_{i},
\end{aligned}
$$

where

$$
u_{i}^{*}(t)=\frac{-\beta A_{i}(t)+C_{i}(t)}{Y_{i}^{\mathrm{s}}(t)}
$$

a

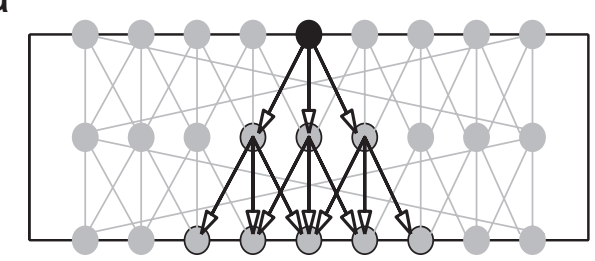

b

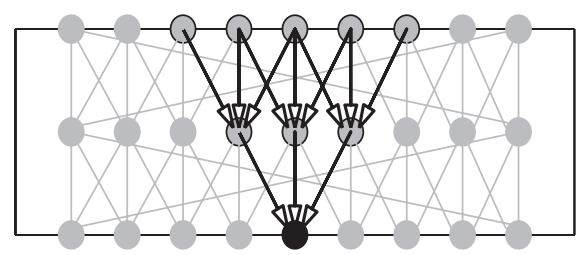

C

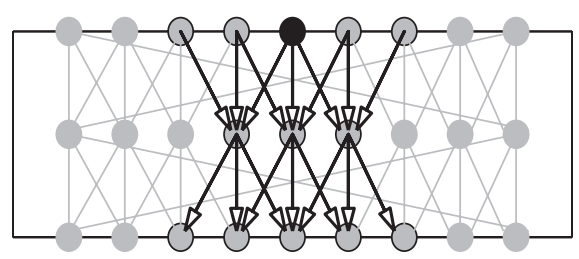

d

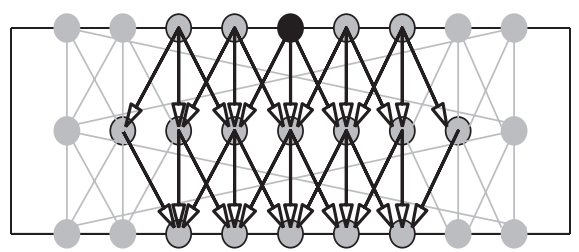

Fig. 2. Different modalities of failure propagation. Edges through which failure propagate are in darker gray. The firm triggering the avalanche is represented by the node in dark gray. (a,b) Downward and upward propagation of failures. (c,d) Horizontal propagation occurs when each level transmits downward but also reflects upwards. In panel $\mathrm{c}$ failures have propagated up to two degrees of separation from the initial firm; in panel d up to three degrees. 
is the critical value of price below which firm $i$ goes bankrupt. If $\mu\left(u_{i}\right)$ is, for instance, a uniform distribution in $\left[1-\delta_{P}, 1+\delta_{P}\right]$, Eq. (26) yields

$$
\mathscr{P}_{i}^{\mathrm{B}}=\frac{u_{i}^{*}(t)+\delta_{P}-1}{2 \delta_{P}} .
$$

Notice that, as expected, $\mathscr{P}_{i}^{\mathrm{B}}=0$ if $u_{i}^{*}=1-\delta$ and that $\mathscr{P}_{i}^{\mathrm{B}}$ increases when the cost $C_{i}$ increases, or when the net worth $A_{i}$ decreases, or finally when paid output $Y_{i}^{\mathrm{s}}$ decreases.

In a regime in which supply equals demand, and neglecting fluctuations of supply prices, we can write the critical price for bankruptcy as

$$
u_{i}^{*}(t)=\frac{-\beta A_{i}(t)+C_{i}(t)}{Y_{i}^{\mathrm{s}}(t)} \simeq \frac{\left(c_{\mathrm{r}}-\beta / \theta\right) Y_{i}^{\mathrm{e}}(t)+c_{\mathrm{s}} Y_{i}(t)}{Y_{i}^{\mathrm{s}}(t)} .
$$

From the equation above we can deduce some conditions under which the bankruptcy (and in general the failure of supply and payments) of a firm affects the probability that a connected firm goes bankrupt.

Notice that if payments are delayed, we cannot replace $Y_{i}^{\mathrm{s}}(t)$ with $Y_{i}(t)$ in the equation, because if some customers go bankrupt at time $t$, then $Y_{i}^{\mathrm{s}}(t)<Y_{i}(t)$. Moreover, if firms cannot adapt the cost for resources within one time step (as assumed in the model), we cannot replace $Y_{i}^{\mathrm{e}}(t)$ with $Y_{i}(t)$, because in case of supply failure it holds $Y_{i}^{\mathrm{e}}(t)>Y_{i}(t)$. Therefore, in this case, the probability that firm $i$ will go bankrupt is affected both by bankruptcies of customers as well as suppliers, and bankruptcies can propagate both upward and downward.

In contrast, if payments are anticipated and firms can instantly adapt their resources to the input received, then the simplifications mentioned above are legitimate and Eq. (28) becomes

$$
u_{i}^{*}(t)=\left(c_{\mathrm{r}}-\beta / \theta\right)+c_{\mathrm{s}}
$$

which implies that the probability of bankruptcy for firm $i$ is a constant, and in particular it is independent of what happens to suppliers and customers. In this case there is no propagation of bankruptcies at all.

As intermediate situations, we have:

1. If payments are delayed but firms are adaptive within one period, then firms are only sensitive to bankruptcies of customers and therefore bankruptcies can propagate only upward.

2. If payments are anticipated but firms are not adaptive within one period, then firms are only sensitive to bankruptcies of suppliers and therefore bankruptcies can propagate only downwards.

Finally, let us consider Eq. (29) in the case of fixed prices $u_{i}(t)=1 \forall i$, , which implies that all firms go bankrupt if

$$
1<\left(c_{\mathrm{r}}-\beta / \theta\right)+c_{\mathrm{s}} .
$$




\subsection{Remarks on the role of the network structure}

Within the scope of the decision making scenarios investigated in this paper, the effect of the network structure can be predicted very simply, yet such scenarios are still too simplified to offer interesting insights for policy. For instance, in scenario 1, firms would benefit from a high connectivity degree (the number of outgoing and ingoing edges in the graph of Fig. 1). This is because the loss of profit due to the bankruptcy of a supplier or a customer is of order of $1 / k_{i}$, where $k_{i}$ is the connectivity degree of firm $i$. In particular, for any set of values of the parameters $\beta, \theta, c_{\mathrm{s}}, c_{\mathrm{r}}$, the connectivity degree could be set large enough to reduce arbitrarily close to zero the probability that a bankruptcy could induce other bankruptcies. In scenario 2, firms would always end up with one main supplier, no matter how many they are possibly connected to. But they would also benefit from a high connectivity degree because in case of the bankruptcy of a supplier, they would have better chances of being able to re-allocate their order to a supplier with a large enough production capacity. The investigation of the role of the structure of the network on the propagation of bankruptcies is left for further developments which will include more realistic order allocation strategies.

\section{Analysis of simulation results}

We now present the results of computer simulations of the model described above with the following specifications which are valid for all the simulations, unless otherwise mentioned. The structure of the network is the one illustrated in Fig. 1, with three suppliers and three customers per firm. Price distribution is uniform in the interval $\left[1-\delta_{P}, 1+\delta_{P}\right]$. We are interested in a regime in which firms' profit is sensitive to unexpected variations of input from suppliers. For this to be, profit variations due to stochastic price fluctuation must not be larger than those due to the variation of input, that is, $\delta_{P}$ must be relatively small. The generic features displayed by the model include:

1. Spatio-temporal correlation of output, growth and bankruptcies.

2. Exponential growth.

3. Oscillations of de-trended aggregate output.

4. Heterogeneous firm size distribution.

5. Exponential probability distribution of aggregate growth (right side).

As mentioned in Section 2.8, in this paper we consider two simple scenarios for the decision-making process of the firm. The model displays the same general features in the two scenarios, but, as discussed in Section 4.2, the strategy for order allocation affects the bankruptcy avalanches, as well as the distribution of output and aggregate growth. On the other hand, introducing a varying interest rate also has a very significant impact on the dynamics of the system, as discussed in Section 4.3. 


\subsection{Parameter settings}

The parameters of the model can be grouped as follows.

1. Size of the network and time span: number of firms $N$, number of levels $M$ in the economy, number of time steps $T$. We test $M$ between 3 and 5, a range which is close to real production networks. We have performed simulations with $N$ of the order of a thousand and $T$ up to a few thousands.

2. Prices, production failure and costs: price interval width $\delta_{P}$, production failure probability $q$, supply cost factor $c_{\mathrm{s}}$. Unless specified otherwise, results reported in this paper are obtained with $\delta_{P}=0.2, q=0.02, c_{\mathrm{s}}=c_{\mathrm{r}}=0.35$.

3. Bankruptcy and rebirth: inactivity time is set as $\tau=3$. Firms are initially all endowed with the same value of net worth $A_{i}(0)=A_{\text {init }}=1 \forall i$ and output $Y_{i}(0)=Y_{\text {init }}=1 \forall i$. Because the total level of net worth is not constant (the economy grows or recedes depending on the parameters at point 2), the entry level of net worth $A_{\text {entry }}$ is set to be proportional to the average value of net worth across firms, $\bar{A}: A_{\text {entry }}=10^{-1} \bar{A}$. The bankruptcy threshold is set as $\beta=0.2$. The depreciation factor is set as $\rho=0.97$ (which yields a depreciation rate $1-\rho=3 \%)$.

\subsection{Scenarios $1-2$}

In a first set of simulations (scenario 1) firms are not able to adapt their resources when a supplier goes bankrupt. Both the structure and the intensity of the connections are static in this case. Correspondingly, for allocation of orders, they use strategy 1 , which is blind to the production capacity of the suppliers and also to their bankruptcies, which means that firms keep allocating one-third of their orders to each supplier regardless of its activity status.

In a second set of simulations (scenario 2), firms can adapt their resources when a supplier goes bankrupt, so they do not incur costs during the inactivity time of the supplier. Moreover, firms adapt orders to the production capacity of the suppliers (strategy 2 for order allocation), which implies that although the structure of the network is still static, the intensity of the links is now changing over time. In both sets of simulations parameters are set as in Section 4.1.

One of the main features of this model is the emergence of correlations in time between output and growth of connected firms. In this respect, it is more illustrative to display examples of the emerging patterns, rather than the plot of the correlation function. In Fig. 3, the evolution of output over the production network in scenario 1 is shown in an interval of 20 time steps. Each frame represents the production network at a given time step. Primary producers are in the leftmost level of each frame. Retailers in the consumer market are in the rightmost level of each frame. Output is normalized by the maximum value of output at each time step in order to emphasize the relative spatial distribution of output. Output is represented by a gray scale as specified by the color bar. Dark gray represents output close to 0 while black represents bankruptcy. Firms have a uniform distribution of output at time 0 , but 


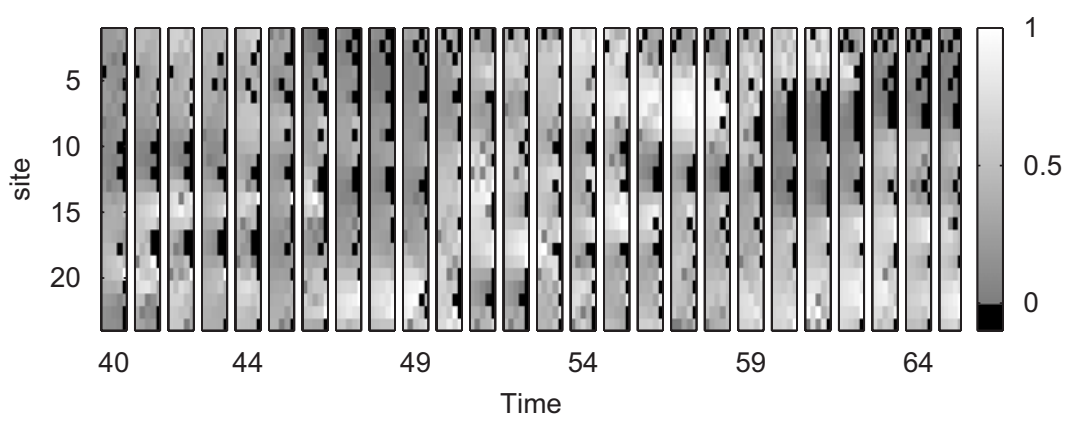

Fig. 3. Time evolution of output of the production network. Zoom on a time interval and in a region of the network. Each column represents the production network at a given time step: the $x$-axis represents production level while the $y$-axis represents firm's position in the network. Primary producers are in the leftmost side of each column. Retailers on the consumer market are in the rightmost side of each column. Output is normalized by the maximum value of the output at each time step and represented with a gray scale. Dark gray represents output close to 0 while black represents bankruptcy. Propagation of failures is observed (see text for more details).

soon regions of high output emerge while other collapse. In particular, following the position of the black cells from one frame to the next, it is possible to observe the propagation of bankruptcies over time. For instance, at time 40, the bankruptcy of firm in position 18 of the third level induces the bankruptcy at time 41 of some customer firms (positions 19,18,17 in the fourth level). A little avalanche is triggered and propagates perpendicularly to the direction of production), until it overlaps at time 46 with another avalanche which at time 40 was concerning firms 9-13.

When firms' orders are adaptive (scenario 2) bankruptcy avalanches tend to be smaller (Fig. 4) and output tend to be concentrated in thinner channels. Adaptive orders result in firms relaying mostly on one supplier.

Similarly to what is shown for the output of the network, Fig. 5 shows a zoom of the time evolution of growth of firms in scenario 1. Growth of firms is measured as $\left(A_{i}(t+1)-A_{i}(t)\right) / A_{i}(t)$. However, for sake of visualization values larger than 0.1 have been set to 0.1 and values smaller than -0.1 have been set to -0.1 . Moreover, bankrupted firms are represented in black. We observe that values of growth of neighboring firms tend to be correlated in time. Interestingly, if we look at the aggregate growth, as defined in Eq. (25), we can observe phases of growth above the average, alternating with phases of growth below the average (Fig. 6).

Growth of aggregate output follows an exponential trend as shown in Fig. 7 for both scenarios 1 and 2 . In the first scenario, growth is smaller than in the second, mainly due to the resource cost that firms incur when suppliers go bankrupt. Moreover, the order allocation strategy implies that except when supplies equal demand, part of the orders are systematically not fulfilled. Strategy 2 for order allocation is not a good strategy, absolutely speaking. It is better than a blind strategy such as strategy 1 , but more sophisticated strategies could be designed and compared. In particular, strategy 2 leads to supply chains where each firm has usually one main supplier, which would be outperformed by a strategy that preserves 


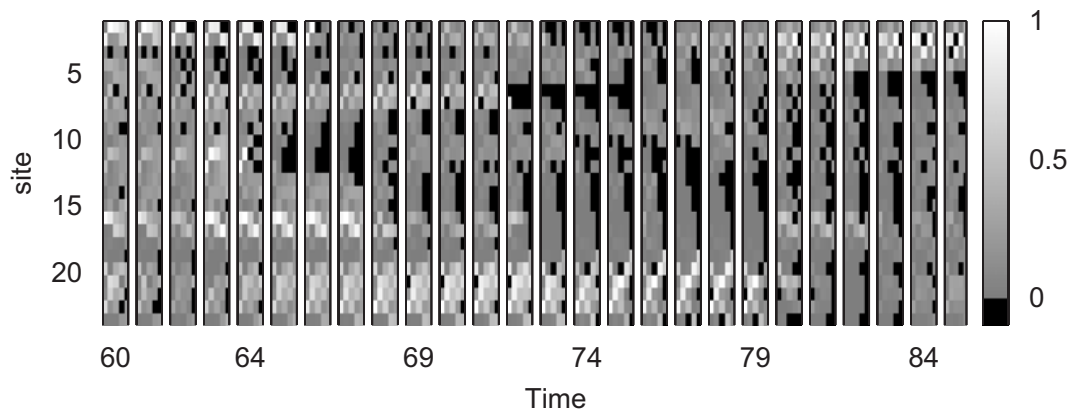

Fig. 4. Time evolution of output of the production network in scenario 2 (strategy 2 for order allocation). The figure is obtained as described in Fig. 3.

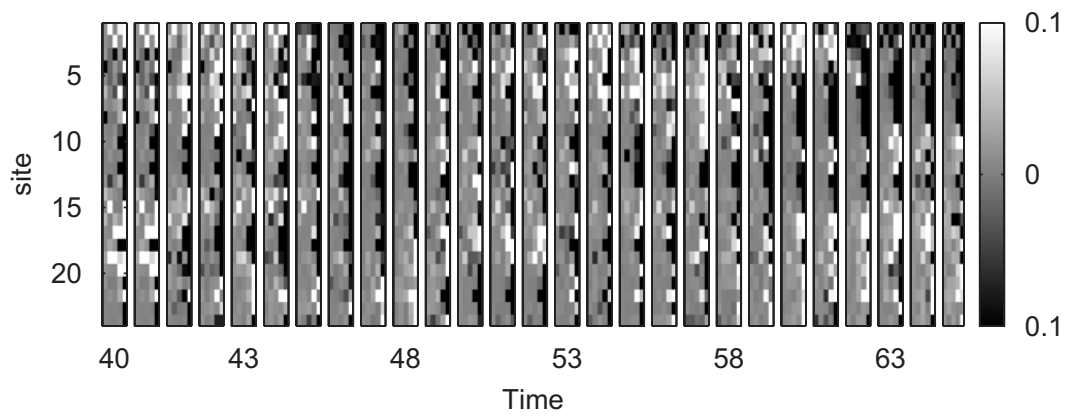

Fig. 5. Time evolution of growth of the production network in scenario 1. Zoom on a time interval. The figure is obtained as described in Fig. 3.

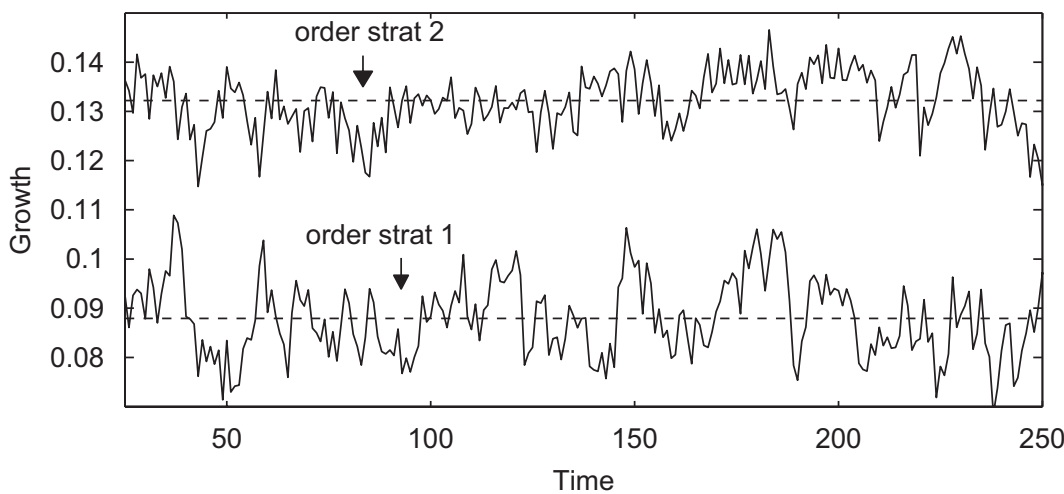

Fig. 6. Time evolution of aggregate growth with strategies 1 and 2 for order allocation. The dashed line represents the average over time. 


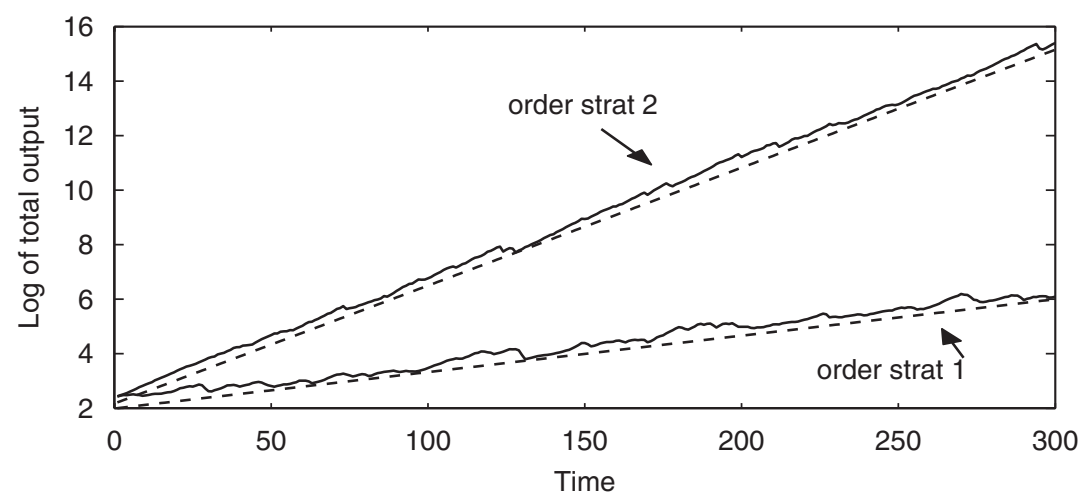

Fig. 7. Time evolution of logarithm in base 10 of aggregate output with strategies 1 and 2 for order allocation.

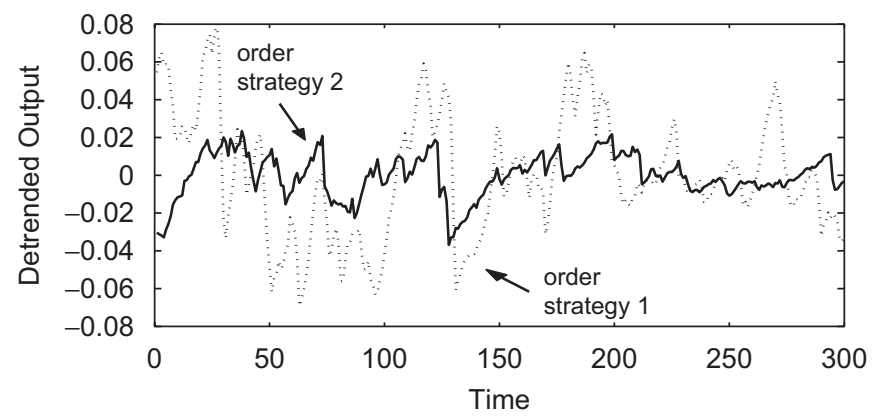

Fig. 8. Time evolution of de-trended aggregate output with strategies 1 and 2 for order allocation (see text).

some diversity in the portfolio of suppliers. However, a more sophisticated strategy for allocating orders requires firms to know how much production capacity is left to each supplier, which in turn requires a non-synchronous allocation of orders and therefore a different setting for the model. The aim of the present work is only to show that order allocation strategies do affect the aggregate output and growth.

The de-trended aggregate output, $Y_{\text {detr }}$, is obtained as the difference between the time series of the logarithm in base 10 of the aggregate output and the linear fit of the time series itself:

$$
Y_{\text {detr }}(t)=\log _{10}\left(Y_{\text {tot }}(t)\right)-b t+a,
$$

where $b$ and $a$ are the coefficients obtained with linear fit. Fig. 8 refers to scenario 2 and shows irregular oscillations of a few percent.

In scenario 2, we find that the right part of the distribution of aggregate growth over time is exponential, while the left part does not display a definite trend (as shown in Fig. 9a). The constant of the exponential decay depends on the parameter setting, but the trend is robust across the parameter range. A similar distribution is 
a

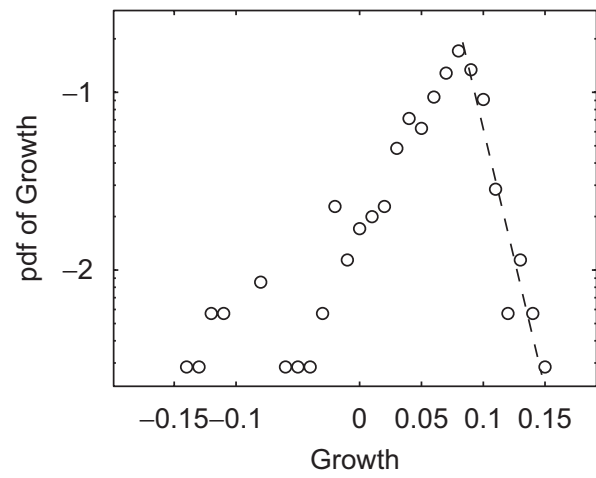

b

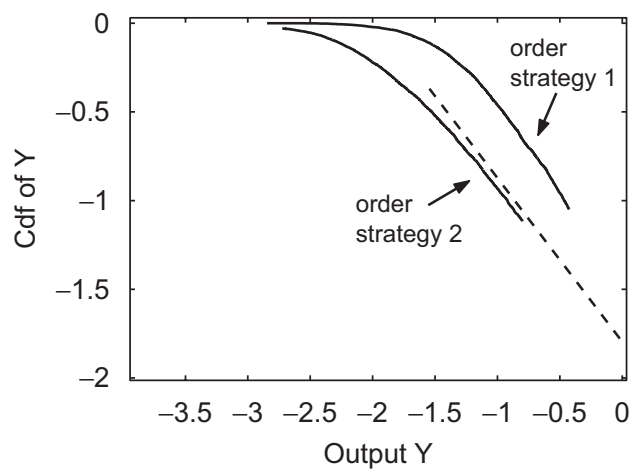

Fig. 9. (a) Probability distribution of growth of aggregate output over time. (b) Complementary cumulative probability distribution of output across firms in the network. Strategy 2 for order allocation gives rise to a power low tail with exponent 2.02. Strategy 1 gives raise to a less heterogeneous distribution.

also found for the distribution of growth across firms (not shown). In contrast, empirical works have found that growth follows a Laplace distribution (Delli Gatti et al., 2005), which is a symmetric exponential distribution. Therefore, some further analytical work is needed to understand to origin of this discrepancy.

The heterogeneity of output across firms is measured by the complementary cumulative distribution. As we observe in Fig. 9b, the order allocation strategy also affects the shape of output distribution. As expected, strategy 1 gives rise to a less heterogeneous distribution than strategy 2. In particular, with strategy 2 the right tail of the distribution follows a power law with exponent 2.02. The value of the exponent, which is consistent with empirical values for size distribution of firms, was found not to be critically sensitive to the range of values of the parameters. A thorough investigation of the dependence of such exponent from the parameters is beyond the scope of this paper.

\subsection{Scenario 3: impact of dynamic interest rate}

We want now to explore, albeit in a very preliminary way, the potential impact of endogenously determined interest rate changes on the dynamics of the system. When a borrowing firm goes bankrupt, the non-performing loan negatively affects the lender's balance sheet. The lender, in turn, could react by pushing up the interest rate charged to other borrowers in an effort to offset the potential loss. In order to capture this effect, we assume that an increase in bankruptcy risk leads to an increase in interest rate and therefore in production costs. Therefore, the production cost for firm $i$ must be multiplied by the factor

$$
1+\eta^{\text {loc }} \frac{\sum_{j \in \text { failed neighb. }} Y_{j}\left(t_{j}^{*}\right)}{\sum_{k \in \text { all neighb. }} Y_{k}(t)}+\eta^{\text {glob }} \frac{\sum_{j \in \text { failed firms }} Y_{j}\left(t_{j}^{*}\right)}{\sum_{k \in \text { all firms }} Y_{k}(t)},
$$




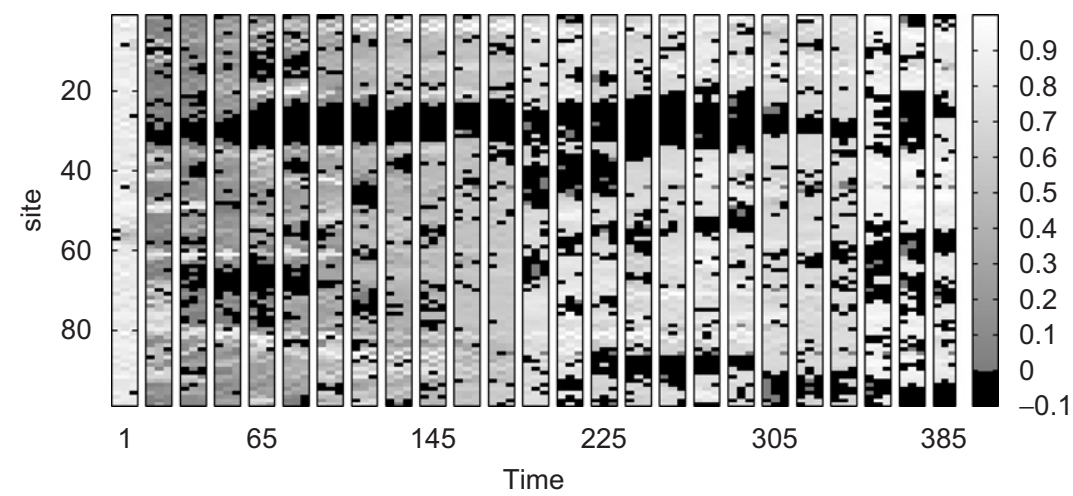

Fig. 10. Time evolution of output of the production network in scenario 3 (strategy 2 for order allocation and dynamic interest rate). The figure is obtained as described in Fig. 3.

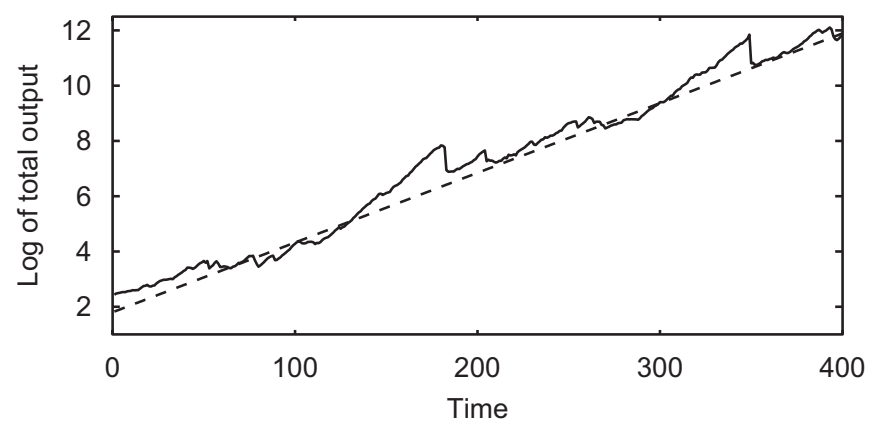

Fig. 11. Time evolution of logarithm in base 10 of aggregate output in scenario 3 .

where parameters $\eta^{\text {loc }}$ and $\eta^{\text {glob }}$ measure the influence of bankruptcies in the neighborhood and in the whole network, respectively. The second term in Eq. (32) represents the fraction of output lost because of the bankruptcies among firms connected to $i . Y_{j}\left(t_{j}^{*}\right)$ is the value of the output of firm $j$ at the moment when it went bankrupt. The third term in the equation concerns the output lost because of bankruptcies everywhere in the network.

We present the results of a third set of simulations (scenario 3) in which the order strategy is as in scenario 2, while production costs are affected, as described above, by bankruptcies in the neighborhood and/or everywhere in the network. In scenario 2 , firms are adaptive and occasional bankruptcies would not give rise to significant avalanches. However, in the presence of a strong enough local and global impact on the interest rate ( $\eta^{\text {loc }}$ and $\eta^{\text {glob }}$ equal 1.25 and 0.75 , respectively), we observe that some bankruptcies trigger local avalanches, which in turn facilitate the onset of bankruptcies elsewhere in the network (Fig. 10).

The aggregate output is still exponentially growing in time, but is characterized by phases of higher and lower growth (Fig. 11), which is also visible in the de-trended 


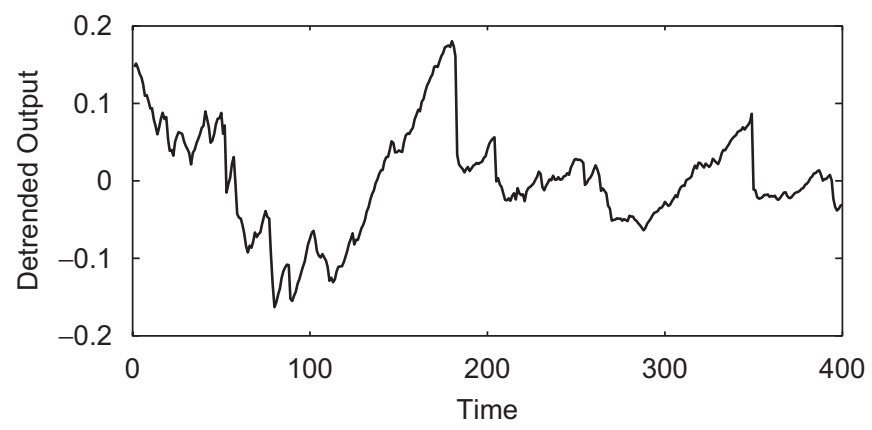

Fig. 12. Time evolution of de-trended aggregate output in scenario 3 .

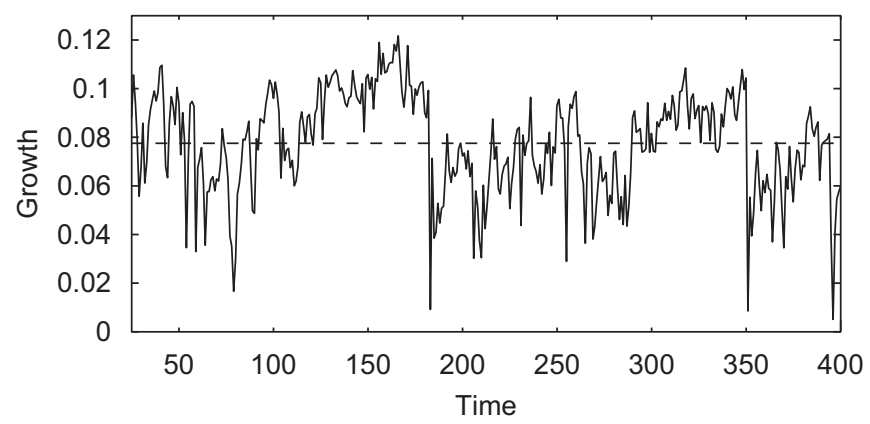

Fig. 13. Time evolution of aggregate growth in scenario 3. The dashed line represents the average over time.

output (Fig. 12) and in the aggregate growth (Fig. 13). Overall, the dynamics of the interest rate induces endogenously the onset of oscillations and instabilities in the system.

\section{Conclusions}

In this paper we have developed a general framework to represent a networked economy in order to explore the effects of local interaction among firms connected by production and credit ties. The framework can yield avalanches of bankruptcies in the presence of delayed payments (trade credit) and costs due to failures in supply. Previous models ignore local interaction so that the propagation of bankruptcies is activated only by means of global coupling: the more firms fail, the higher the interest rate for all, hence the more they fail. Therefore, this paper provides a novel and alternative mechanism for the propagation of failures.

Within the proposed framework, we have implemented and simulated a specific model in which fluctuations of aggregate output - around a trend of exponential growth - emerge from the evolution in space and over time of the local interaction 
among firms. A certain number of stylized facts can also be reproduced in a quite straightforward way. The calibration and validation of the model in the light of real world data is an issue to be dealt with accurately in a follow up of the present paper.

Adjusting the interaction rules assumed in the model, it is possible to investigate the role of the main factors involved in models of financial fragility and address the following issues:

(1) The role of trade-credit relationships in the propagation of bankruptcies.

(2) The role of interest rate and policies to prevent the occurrence of large avalanches.

(3) The role of the structure of the network of interactions.

(4) Policies to make such structure more robust against large avalanches.

In this paper we have started addressing the first two issues. In future developments we will address the third one and further investigate the first two. In conclusion, we believe that this framework opens the way to a novel class of models for endogenous business fluctuations based on firm-firm interaction and trade-credit relationships.

\section{Acknowledgments}

S.B. acknowledges the CNRS fellowship at CAMS, EHESS, 75006 Paris, France, which supported part of this work, as well as the European project MMCOMNET, IST contract no 12999. S.B., D.D.G and M.G. acknowledge the EXYSTENCE Thematic Institute CHIEF (held at Univ. Polit. d.le Marche, Ancona, Italy in May 2005 for supporting this collaboration).

\section{References}

Allen, F., Gale, D., 2000. Financial contagion. Journal of Political Economy 108.

Bak, P., Chen, K., Scheinkman, J., Woodford, M., 1993. Aggregate fluctuations from independent sectoral shocks: self-organized criticality in a model of production and inventory dynamics. Ricerche Economiche 47, 3-30.

Biham, O., Malcai, O., Levy, M., Solomon, S., 1998. Generic emergence of power law distributions and Lévy-stable intermittent fluctuations in discrete logistic systems. Physical Reviews E 58, 1352 〈http:// xxx.lanl.gov/abs/adap-org/9804001>.

Boissay, F., 2006. Credit chains and the propagation of financial distress. European Central Bank Working Paper, 573.

Delli Gatti, D., Di Guilmi, C., Gaffeo, E., Giulioni, G., Gallegati, M., Palestrini, A., 2005. A new approach to business fluctuations: heterogeneous interacting agents, scaling laws and financial fragility. Journal of Economic Behavior and Organization 56 (4), 489-512 〈http://arxiv.org/abs/cond-mat/0312096〉.

Elligsen, T., Burkart, M., 2002. In-kind finance. LSE Financial Markets Group Discussion Paper, 421.

Greenwald, B., Stiglitz, J., 1993. Financial market imperfections and business cycles. Quarterly Journal of Economics 108, 77-114.

Hahn, F.H., Solow, R., 1995. A Critical Essay on Modern Macroeconomic Theory. MIT Press, Cambridge, MA. 
Kesten, H., 1973. Random difference equations and renewal theory for products of random matrices. Acta Mathematica 131, 207-248.

Kiyotaki, N., Moore, J., 1997. Credit cycles. Journal of Political Economy 105, 211-248.

Klapper, L., 2001. The uniqueness of short-term collateralization. World Bank, Policy Research Working Paper, 2544.

Lee, Y., Stowe, J., 1993. Product risk, asymmetric information and trade credit. Journal of Financial and Quantitative Analysis 28.

Leijonhuvfud, A., 1992. Keynesian economics: past confusions, future prospects. In: Vercelli, A., Dimitri, N. (Eds.), Macroeconomics: A Survey of Research Strategies. Oxford University Press, Oxford, pp. 16-37.

Leijonhuvfud, A., 1993. Towards a not-too-rational macroeconomics. Southern Economic Journal 60, $1-13$.

Nirei, M., Souma, W., 2003. Income distribution and stochastic multiplicative process with reset events. In: The Complex Dynamics of Economic Interaction. Springer, Berlin.

Omiccioli, M., 2005. Trade credit as collateral. Temi di discussione della Banca d'Italia, 553.

Petersen, M., Rajan, R., 1997. Trade credit: theories and evidence. Review of Financial Studies 10.

Sornette, D., 1998. Multiplicative processes and power laws. Physical Reviews E 57 (N4), 4811-4813.

Stiglitz, J., Greenwald, B., 2003. Towards a New Paradigm in Monetary Economics. Cambridge University Press, Cambridge, UK.

Weisbuch, G., Battiston, S., 2007. From production networks to geographical economics. Journal of Economic Behavior and Organization, forthcoming.

\section{Further Reading}

Kirman, A., 1992. Whom or what does the representative individual represents? Journal of Economic Perspectives 6, 117-136. 